Math. Ann. 311, 27-40 (1998)

Mathematische

Annalen

(c) Springer-Verlag 1998

\title{
Fatou and Julia sets for entire mappings in $\mathbb{C}^{k}$
}

\author{
John Erik Fornæss ${ }^{1, \star}$, Nessim Sibony ${ }^{2}$ \\ 1 Mathematics Department, The University of Michigan, Ann Arbor, MI 48109, USA \\ ${ }^{2}$ Université Paris Sud, URA757, Bat 425. Mathématiques, F-91405 Orsay, France
}

Received: 3 June 1996 / Revised version: 17 March 1997

Mathematics Subject Classification (1991): 32H50, 58F23

\section{Introduction}

It is a quite natural idea to extend the Fatou-Julia theory of iteration of holomorphic maps in $\mathbb{C}, \overline{\mathbb{C}}$ to higher dimension. One can even restrict to interesting classes of holomorphic maps, like polynomial automorphisms of $\mathbb{C}^{2}$, biholomorphic maps of $\mathbb{C}^{k}$, volume preserving biholomorphic maps etc. The special case of (polynomial) Hénon maps in $\mathbb{C}^{2}$ has been extensively studied. (See ([FSO]) for references.)

Here the emphasis is on transcendental self maps of $\mathbb{C}^{k}$.

Given $\Phi: \mathbb{C}^{k} \rightarrow \mathbb{C}^{k}$, a holomorphic self map of generic rank $k$, we define the Fatou set $\mathscr{T}_{\Phi}$ as the open set of normality of $\left(\Phi^{n}\right)_{n \geq 0}$. (We allow $\infty$ as a limit.) The complement, $\mathscr{J}_{\Phi}$, is the Julia set.

It was proved in ([FS4]) that for a dense $G_{\delta}$ set in the group $\mathscr{B}$ of biholomorphic maps in $\mathbb{C}^{k}$ the Fatou set is just made of basins of attraction corresponding to attracting cycles and that hyperbolic periodic cycles are dense in the Julia set. Similar results are obtained in ([FS3]), ([FS4]) for other classes of mappings, see Theorems 2.9 and 2.10 below.

In $([\mathrm{Bu}])$, Buzzard proved a version of the Kupka-Smale Theorem for $\mathscr{B}$.

In this paper we want to study the first properties of Fatou and Julia sets for holomorphic maps of generic maximal rank, $\mathscr{E}_{m}$.

In the second paragraph we give some properties and examples. In the third paragraph we describe recurrent Fatou components for maps in $\mathscr{E}_{m}$.

In paragraph 4 , we show that the Julia set of a map $\Phi \in \mathscr{E}_{m}$ is perfect unless the map is conjugate to an upper triangular automorphism. In the same vein, we

\footnotetext{
* First author supported by an NSF grant.
} 
show that if $\Phi$ is a biholomorphism of $\mathbb{C}^{k}$, not conjugate to an upper triangular automorphism, then the Julia set of $\Phi$ has no compact component.

In the last paragraph we construct a biholomorphism $\Phi$ of $\mathbb{C}^{2}$ with a wandering Fatou component $\Omega$, such that a subsequence $\left(\Phi^{n_{i}}\right)$ converges to $\infty$ on $\Omega$ and another subsequence $\left(\Phi^{m_{i}}\right)$ has a finite limit on $\Omega$; we call such a component an oscillating Fatou component.

\section{Basic definitions}

We let $\mathscr{E}$ denote the space of entire holomorphic maps $\Phi$ on $\mathbb{C}^{k}$ and $\mathscr{E}_{m} \subset \mathscr{E}$ the space of endomorphisms of generic maximal rank $k$. For every map $\Phi \in \mathscr{E}$, $\mathbb{C}^{k}$ is naturally divided in two subsets, the set of normality and its complement. We say that a family of maps is normal if each subsequence has a subsequence which either converges uniformly on compacts or diverges uniformly to infinity.

Definition 2.1 The Fatou set $F=F_{\Phi}$ of a map $\Phi \in \mathscr{E}$, is the largest open set on which the iterates $\Phi^{n}$ is a normal family.

Definition 2.2 The Julia set $J=J_{\Phi}$ consists of the complement of $\mathscr{F}$.

The main problems are to describe the nature of the components of the Fatou set and the structure of the Julia set.

We need the following classical result, which can be found for example in the textbook ([G, p. 137]) by Gunning.

Proposition 2.3 Let $\Phi \in \mathscr{E}_{m}$. There exists a closed analytic set $\Sigma$ contained in the branching locus of $\Phi$ such that no level sets of $\Phi_{\mid \Sigma}$ has discrete points. Moreover $\Phi$ is open and has discrete fibers on $\mathbb{C}^{k} \backslash \Sigma$.

We call $\Sigma\left(\Sigma_{n}\right)$ the constant set of $\Phi\left(\Phi^{n}\right)$.

Next we state invariance properties for the Fatou and Julia sets. These follow from the definitions and the previous proposition.

Proposition 2.4 Given $\Phi \in \mathscr{E}_{m}$ with Fatou set $F$, Julia set $J$ and constant set $\Sigma$. The following relations hold:

1) $\Phi^{-1}(F) \subset F$,

2) $\Phi(F) \supset F \cap \Phi\left(\mathbb{C}^{k}\right)$,

3) $\Phi\left(F \cap \Sigma^{c}\right) \subset F$, and dually,

4) $\Phi^{-1}(J) \supset J$

5) $\Phi(J) \subset J\left(=J \cap \Phi^{-1}\left(\mathbb{C}^{k}\right)\right)$ and

6) $\Phi^{-1}(J) \cap \Sigma^{c} \subset J$.

We can strengthen 3) for some Fatou components.

Proposition 2.5 Let $\Omega$ be a Fatou component for $\Phi \in \mathscr{E}_{m}$. Assume $\left\{\Phi^{n}\right\}$ is locally bounded on $\Omega$. Then $\Phi(\Omega)$ is contained in the Fatou set. 
Proof. Recall at first that for a compact set $K \subset \mathbb{C}^{k}$ the polynomially convex hull $\hat{K}$ of $K$ is defined by

$$
\hat{K}=\left\{z \in \mathbb{C}^{k} ;|P(z)| \leq \sup _{K}|P| \forall \text { holomorphic polynomials } P\right\} .
$$

A domain $U$ is Runge if $K \subset \subset U$ implies $\hat{K} \subset \subset U$. Here $K$ may be assumed to be connected and $\hat{K}$ is then automatically connected ([Hö]). We show first that $\Omega$ is Runge. Let $K \subset \subset \Omega$. Let $K_{\delta}=\{z ; \operatorname{dist}(z, K) \leq \delta\}$. Choose $\delta>0$ so that $K_{\delta} \subset \subset \Omega$. It is clear that $\left\{\Phi^{n}\right\}$ is uniformly bounded on $\hat{K}_{\delta}$ and it is easy to check that $\hat{K}_{\delta}$ contains a neighborhood of $\hat{K}$. Hence $\Omega$ is Runge. Similarly, $\Phi(\Omega \backslash \Sigma)$ is contained in a Runge Fatou component $\Omega^{\prime}$ since $\Phi$ is open on $\Omega \backslash \Sigma$. Let $p \in \Omega \cap \Sigma$. Pick a disc $\Delta$ centered at $p$ and such that $\bar{\Delta} \backslash p \subset \Omega \backslash \Sigma$. Then $\Phi(\bar{\Delta} \backslash p) \subset \Omega^{\prime}$, hence $\Phi(p) \in \Omega^{\prime}$, using that $\Omega^{\prime}$ is Runge.

Example 2.6 Let $f(z)=\lambda e^{z}, z \in \mathbb{C}, 0<\lambda<e^{-1}$. Then the Fatou set has one single component $\Omega$ containing 0 , but $0 \notin f(\Omega)$. So assertion 3) in Proposition 2.4 is sharp even when $k=1$. Observe that when $k=1, \Sigma$ is empty if $\Phi$ is not constant. The example is mentioned in ([Be], p. 162)

Examples 2.7 i) $\Phi: \mathbb{C}^{2} \rightarrow \mathbb{C}^{2}:(z, w) \rightarrow(z w, z)$.

Then $\Phi^{n}(z, w)=\left(z^{p_{n}} w^{p_{n-1}}, z^{p_{n-1}} w^{p_{n-2}}\right), n \geq 2, p_{0}=p_{1}=1, p_{n+1}=$ $p_{n}+p_{n-1}, \quad n \geq 1$. Then $p_{n}=\frac{1}{\sqrt{5}}\left(\alpha^{n+1}-\beta^{n+1}\right), \alpha=\frac{1+\sqrt{5}}{2} \beta=\frac{1-\sqrt{5}}{2}$. $\left|Z_{n}\right|:=\left|z^{p_{n}} w^{p_{n-1}}\right|=\left|z w^{\frac{p_{n-1}}{p_{n}}}\right|^{p_{n}}$, with $\frac{p_{n-1}}{p_{n}} \rightarrow \frac{1}{\alpha}$. If $|z||w|^{\frac{1}{\alpha}}>1, Z_{n} \rightarrow \infty$. If $|z||w|^{\frac{1}{\alpha}}<1, Z_{n} \rightarrow 0$. The Julia set, $\left\{|z||w|^{\frac{1}{\alpha}}=1\right\}$, is smooth.

The Fatou set has two components, the domain of attraction of 0 and the domain of attraction of infinity. Observe that in this case $\Sigma=\{z=0\}$ is in the Fatou set. Periodic points other than $(0,0)$ are roots of unity on $\{|z|=|w|=1\}$ and they are hyperbolic. The restriction of $\Phi^{2}$ to the torus $\{|z|=|w|=1\}$ is a linear hyperbolic map. Periodic points are dense. It is easy to check that the Julia set is foliated by the corresponding stable leaves.

ii) We now consider an example of a biholomorphic map of $\mathbb{C}^{2}$. Let $h$ be an entire function of one variable. Define $\Phi(z, w)=\left(z e^{h(z w)}, w e^{-h(z w)}\right)$, then $Z_{1} W_{1}=z w$ so on $z w=\alpha, \Phi^{n}(z, w)=\left(z e^{n h(\alpha)}, w e^{-n h(\alpha)}\right)$. If $\Re h(0)<0$, then $J=\{\Re h(z w)=0\} \cup\{w=0\}$. If $h(\zeta)=\zeta-1 / 2, J$ is not connected. The domain of attraction of infinity is pseudoconvex but not Runge. The variety $\{w=0\}$ is the stable manifold of $(0,0)$, it's contained in $J$ and not dense in $J$. Observe that points in $J$ have bounded orbit.

If $\Re h(0)=0$ then $J=\{\Re h(z w)=0\}$. The varieties defined by $h(z w)=\frac{2 i k \pi}{n}$ are fixed by $\Phi^{n}$. Their union is dense in J. The Fatou set can have infinitely many components, all of them in the basin of attraction of infinity.

Proposition 2.8 Let $\Omega$ be a Fatou component for $\Phi \in \mathscr{E}_{m}$. Let $\Omega^{\prime}$ be the Fatou component containing $\Phi(\Omega \backslash \Sigma)$. Then $\Phi(\Omega) \subset \overline{\Omega^{\prime}}$ and $\Phi(\partial \Omega) \subset \partial \Omega^{\prime}$. In particular if $\Phi$ is proper, then $\Phi(\Omega)=\Omega^{\prime}$. 
Proof. We know that $\Phi(\Omega \backslash \Sigma)$ is in the Fatou set. Since $\Omega \backslash \Sigma$ is connected it follows that $\Phi(\Omega \backslash \Sigma)$ is contained in a component $\Omega^{\prime}$ of the Fatou set. The continuity of $\Phi$ implies that $\Phi(\Omega) \subset \overline{\Omega^{\prime}}$.

We show next that $\Phi(\partial \Omega) \subset \partial \Omega^{\prime}$. Assume $p \in \Omega^{\prime} \backslash \Phi(\Omega)$ and $p=\Phi(q)$ with $q \in \partial \Omega$, then $\left(\Phi^{n}\right)$ is normal in a neighborhood of $q$, a contradiction. If $\Phi$ is proper, then $\Phi$ is open so $\Phi(\Omega) \subset \Omega^{\prime}$, and $\Phi(\Omega)$ has no boundary point in $\Omega^{\prime}$.

We introduce some interesting classes of holomorphic endomorphisms of $\mathbb{C}^{k}$. $\mathscr{B}$ : The group of biholomorphic maps.

$\mathscr{T}$ : The group of volume preserving biholomorphic maps.

$\mathscr{P}_{m}$ : The proper holomorphic maps.

These spaces are endowed with the topology of uniform convergence on compact sets. The spaces $\mathscr{B}$ and $\mathscr{T}$ are Baire spaces. Some partial results concerning dynamics of generic maps for $\mathscr{B}, \mathscr{T}, \mathscr{E}$ have been obtained in ([FS2]), ([FS3]) and ([FS4]).

A point $p$ belongs to $\Omega(f)$, the nonwandering set of $f$, if and only if for every neighborhood $U(p)$ there exists $n \geq 1$ such that $f^{n}(U) \cap U \neq \emptyset$.

Theorem 2.9 ([FS4]) There exists a $G_{\delta}$ dense set $\mathscr{V}^{\prime} \subset \mathscr{V}$ such that for every $f \in \mathscr{V}^{\prime}$, hyperbolic periodic orbits are dense in $\mathbb{C}^{k}$, hence the Julia set of $f$, $J_{f}=\mathbb{C}^{k}$. Moreover for a $G_{\delta}$ dense set of points in $\mathbb{C}^{k}$, orbits are dense.

Theorem 2.10 ([FS4]) Let $\mathscr{D}$ denote $\mathscr{B}$ or $\mathscr{E}$. There exists $\mathscr{D}{ }^{\prime} \subset \mathscr{D}$, a dense $G_{\delta}$ set such that, for $f \in \mathscr{D}^{\prime}$ the Fatou set is the union of components (pre)periodic to attracting basins. Moreover for $f \in \mathscr{D}^{\prime}, \Omega(f)$ is the closure of the set of periodic points.

Proposition 2.11 Let $\Phi \in \mathscr{P}$. Then $F_{\Phi}=F_{\Phi^{\ell}}$ and $J_{\Phi}=J_{\Phi^{\ell}}$.

Proof. It is clear that $F_{\Phi} \subset F_{\Phi^{\ell}}$. Let $\Omega$ be a Fatou component for $\Phi^{\ell}:=\Psi$. Let $\left(\Phi^{n_{i}}\right)$ be a subsequence of iterates. Then $n_{i}=m_{i} \ell+r_{i}, 0 \leq r_{i}<\ell$.

Without loss of generality we can assume that for all $i, r_{i}=r, 0<r<\ell$. If $\Psi^{m_{i}}$ has a finite limit then necessarily $\Phi^{r}\left(\Psi^{m_{i}}\right)$ is locally bounded. So assume $\Psi^{m_{i}} \rightarrow \infty$. Then $\Phi^{r}\left(\Psi^{m_{i}}\right) \rightarrow \infty$. Hence $F_{\Phi} \supset F_{\Phi^{\ell}}$, so $F_{\Phi}=F_{\Phi^{\ell}}$. Therefore, by taking complement, $\mathscr{J}_{\Phi}=\mathscr{J}_{\Phi^{\ell}}$ also.

\section{Recurrent Fatou components for $\mathscr{E}_{m}$}

Definition 3.1 Let $\Phi \in \mathscr{E}_{m}$. A Fatou component $\Omega$ is periodic if for some $m>0$, $\Phi^{m}(\bar{\Omega}) \subset \bar{\Omega}$. Equivalently $\Phi^{m}\left(\Omega \backslash \Sigma_{m}\right) \subset \Omega$. A Fatou component $\Omega$ is recurrent if there is a point $q \in \Omega$, and a sequence of integers $n_{j}$, such that $\Phi^{n_{j}}(q) \rightarrow p_{0} \in \Omega$. A Fatou component $\Omega$ is wandering if $\left\{\Phi^{m}\left(\Omega \backslash \Sigma_{m}\right)\right\}$ are pairwise disjoint.

Observe that recurrent Fatou components are perodic.

Because of normality of iterates on Fatou sets this definition is equivalent to 
Lemma 3.2 A Fatou component $\Omega$ is recurrent if and only if there is a compact $K \subset \Omega$, and a sequence of integers $n_{j}$ and points $q_{j} \in K$, such that $\Phi^{n_{j}}\left(q_{j}\right) \rightarrow$ $p_{0} \in \Omega$.

Theorem 3.3 Let $\Phi \in \mathscr{E}_{m}$, the space of entire maps of generic maximal rank. Assume $\Omega$ is a recurrent Fatou component for $\Phi$. Then $\left(\Phi^{n}\right)$ is locally bounded on $\Omega$ and $\Omega$ is pseudoconvex and Runge. Moreover there is a closed connected complex submanifold $S^{\prime} \subset \Omega$ of dimension $r, 0 \leq r \leq k$ and an integer $\ell \geq 1$ such that $\Phi^{\ell}$ is an automorphism of $S^{\prime}$ and $\left(\Phi_{\mid S^{\prime}}^{\ell n}\right)_{n}$ is relatively compact in $\mathrm{Aut}\left(S^{\prime}\right)$. If $\in \overline{\left\{\Phi_{\mid \Omega}^{n}\right\}} \backslash\left\{\Phi_{\mid \Omega}^{n}\right\}$ then $f$ is of generic rank equal to $r$.

Proof. We use the notation of Lemma 3.2. Let $\ell \geq 1$ be the smallest integer for which $\Phi^{\ell}(\Omega) \cap \Omega \neq \emptyset$. Then $\Omega \subset \Omega^{\prime}$, a Fatou component for $\Phi^{\ell}$. We will show that $\Omega^{\prime}$ is recurrent for $\Psi:=\Phi^{\ell}$. Indeed, $\Psi(\Omega) \subset \bar{\Omega}$ so $\Psi^{m}(\Omega) \subset \Psi\left(\Psi^{m-1}(\Omega)\right) \subset$ $\bar{\Omega}$ by induction.

Assume that for some $0<r<\ell, \Phi^{m \ell+r}(\Omega) \cap \Omega \neq \emptyset$. Then $\emptyset \neq \Phi^{r}\left(\Phi^{m \ell}(\Omega)\right) \cap$ $\Omega \subset \Phi^{r}(\bar{\Omega}) \cap \Omega$, hence $\Phi^{r}(\Omega) \cap \Omega \neq \emptyset$, a contradiction. Hence the $n_{j}^{\prime} s$ are multiples of $\ell, n_{j}=m_{j} \ell$, so $\Omega^{\prime}$ is recurrent for $\Psi$. Notice that by Proposition 2.4, $\Psi\left(\partial \Omega^{\prime}\right) \subset \partial \Omega^{\prime}$. We can assume the existence of $q \in \Omega^{\prime}$ such that $\Psi^{m_{j}}(q) \rightarrow$ $p_{0} \in \Omega^{\prime}$.

Consider the set of all maps $h: \Omega^{\prime} \rightarrow \overline{\Omega^{\prime}}$ with $h(p)=p$ for some $p \in \Omega^{\prime}$ and $h=\lim \Psi^{k_{j}}$ for some sequence $k_{j}$. This set is non empty since $\Psi^{m_{j+1}-m_{j}}\left(\Psi^{m_{j}}\right)=$ $\Psi^{m_{j+1}}$, so if $h$ is a limit of a subsequence of $\Psi^{m_{j+1}-m_{j}}$ we will have that $h\left(p_{0}\right)=p_{0}$. Among the maps $h$, fix one of maximal generic rank $r$. If $r=0$, then $h\left(\Omega^{\prime}\right)=p$. We have $\Psi(p)=\Psi(h(p))=\Psi\left(\lim \Psi^{m_{j}}(p)\right)=\lim \Psi^{m_{j}}(\Psi(p))=h(\Psi(p))=p$. $\left(\Psi(p) \in \Omega^{\prime}\right.$ since $h(p)=p$ and $\Psi\left(\partial \Omega^{\prime}\right) \subset \partial \Omega^{\prime}$.)

So $p$ is fixed for $\Psi$ and it is necessarily attracting, hence $\Psi^{n}$ converges u.c.c. to $p$. Hence $p$ belongs to an attracting periodic cycle for $\Phi$. The theorem is proved in this case.

Assume $r \geq 1$. Define $\Sigma=h\left(\Omega^{\prime}\right)$ for some $h$ of maximal rank $r$. We show that $\Psi\left(\Sigma \cap \Omega^{\prime}\right) \subset \Sigma \cap \Omega^{\prime}$. Let $y \in \Sigma \cap \Omega^{\prime}$. Write $y=h(x)$ with $x \in \Omega^{\prime}$. We show first that $\Psi(y) \in \Omega^{\prime}$. Since $\Psi^{m_{i}}(x) \rightarrow y, \Psi^{m_{i+1}-m_{i}}(y) \rightarrow y$. Hence $\Psi(y)$ must be in $\Omega^{\prime}$. Then $\Psi(y)=\Psi(h(x))=\Psi\left(\lim \Psi^{m_{i}}(x)\right)=h(\Psi(x))$ since $\Psi(x) \in \Omega^{\prime}$. So $\Psi(y) \in \Sigma$.

By choosing another $h$ of maximal generic rank $r$ we want to arrange that $h$ is the identity map on some nonempty piece of an $r$ - dimensional manifold.

Let $\Delta$ be a small polydisc centered at $p$. Choosing a suitable small polydisc $\Delta^{\prime} \subset \Delta$ we have that $\sigma:=h\left(\Delta^{\prime}\right)$ is a smooth $r$-dimensional manifold in $\Delta$. Taking a limit $\tilde{h}$ of $\Psi^{m_{i+1}-m_{i}}$ we get that $\tilde{h}=\operatorname{Id}$ on $\sigma$.

Define $S:=\left\{q \in \Omega^{\prime} ; \tilde{h}(q)=q\right\}$. Then $S$ has dimension $\leq r$. Using that $\mathrm{I} d-\tilde{h}$ has rank at least $k-r$, it follows that $S$ is locally contained in a complex manifold of dimension $r$. Hence $S$ is a manifold at each point of dimension $r$. Fix $q_{0} \in \tilde{h}(\sigma) ; \tilde{h}(\sigma) \subset S$ and has dimension $r$. Let $S_{q_{0}}$ be the irreducible component of $S$ containing $\tilde{h}(\sigma)$. Then $S_{q_{0}}$ is a closed submanifold of $\Omega^{\prime}$.

Observe that as above, $\Psi^{j}\left(S_{q_{0}}\right) \subset \Omega^{\prime}$, hence

$\tilde{h} \circ \Psi^{j}=\Psi^{j} \circ \tilde{h}$ on $S_{q_{0}}$. Therefore $\tilde{h}=\operatorname{Id}$ on $\Psi^{j}\left(S_{q_{0}}\right)$. 
Then $\Psi^{j}\left(S_{q_{0}}\right) \subset S$. Since some iterates converge to the identity, for some $i$, $\Psi^{i}\left(S_{q_{0}}\right) \subset S_{q_{0}}$. Since $S_{q_{0}}$ is a manifold and a subsequence of iterates converges to the identity, then $\Psi^{i} \in \operatorname{Aut}\left(S_{q_{0}}\right)$.

Define $Y=\overline{\left\{\Psi_{\mid S_{q_{0}}}^{\text {in }}\right\}_{n}} \cap \operatorname{Aut}\left(S_{q_{0}}\right)$. On $Y$ we use the topology of uniform convergence on compact sets. We want to show that $Y$ is compact. We first show that $Y$ is locally compact.

Fix $g_{0} \in Y$. Let $K$ be a compact set contained in $S_{q_{0}}$. For $\epsilon>0$ small, define

$$
W=\left\{g \in Y ;\left|g-g_{0}\right|_{K}<\epsilon\right\} .
$$

Let $\left\{g_{j}\right\}$ be a subsequence in $W$. We can assume that $g_{j}=\Psi^{i m_{j}}$. Since we are on a Fatou component, $\left\{\Psi^{i m_{j}}\right\}$ has a cluster value, a map $\Theta$ on $\Omega^{\prime}$.

We show that $\Theta\left(S_{q_{0}}\right) \subset S_{q_{0}}$. The classical result on limits of automorphisms uses Kobayashi hyperbolicity. Here we use instead that we are on a Fatou component.

Let $X:=\left\{z \in S_{q_{0}}\right.$; rank $\Theta<r$. $\}$ We first show that $\Theta\left(S_{q_{0}} \backslash X\right) \subset S_{q_{0}}$. Observe that $\Theta^{-1}\left(S_{q_{0}}\right) \cap S_{q_{0}}$ is clearly open in $S_{q_{0}}$ and nonempty. We show this set is closed. Let $x$ be a boundary point of $\Theta^{-1}\left(S_{q_{0}}\right) \cap S_{q_{0}}$ with respect to $S_{q_{0}} \backslash X$ (which is connected). Fix $V$ a neighborhod of $x, \Psi^{i m_{j}}(V)$ are $r$-dimensional manifolds which we can take to be graphs over $\Theta(V)$ (shrinking $V$ a little).

For large $j$, all these graphs have a nonempty set in common with $\Theta(V)$. Hence by the identity theorem $\Theta(V) \subset S_{q_{0}}$.

We show next that $\Theta(X) \subset S_{q_{0}}$. Fix $x \in X$. Let $\Delta$ be a small complex disc in $\left(S_{q_{0}} \backslash X\right) \cup\{x\}$. Let $U$ be a small Runge neighborhood of $\Delta$. For $m_{j}>m_{j_{0}}$ large enough, $\left(\Psi^{i m_{j_{0}}}\right)^{-1} \Psi^{i m_{j}}(\partial \Delta)$ is contained in $U$. Hence by the maximum principle, $\Psi^{i m_{j}}(\Delta) \subset \Psi^{i m_{j 0}}(U) \subset \subset S_{q_{0}}$. Hence $\Theta(x) \in \Theta(\Delta) \subset S_{q_{0}}$. So we have shown that $\Theta\left(S_{q_{0}}\right) \subset S_{q_{0}}$.

We know that some sequence $\Psi^{i \ell} \rightarrow \operatorname{Id}$ on $S_{q_{0}}$. For any $m_{j}$ we can choose $s(j)$ so that $\Psi^{i \ell_{s(j)}-m_{j}}$ is close to the inverse of $\Psi^{i m_{j}}$. Taking a subsequence, we may assume that $\Psi^{i \ell_{s(j)}-m_{j}} \rightarrow \tilde{\Theta}$. As above $\tilde{\Theta}\left(S_{q_{0}}\right) \subset S_{q}$ and $\tilde{\Theta} \circ \Theta=\Theta \circ \tilde{\Theta}=$ Id. Hence $\Theta$ is an automorphism of $S_{q_{0}}$.

Because of the structure of commutative locally compact groups, the existence of a sequence $\Psi^{i m_{j}} \rightarrow$ Id implies that $Y$ is compact. ([Bo])

As a consequence we obtain that $\left\{\Psi^{i m}\right\}$ is locally bounded on $S_{q_{0}}$. Equicontinuity of $\left\{\Psi^{i m}\right\}_{m}$ shows that $\left\{\Psi^{i m}\right\}_{m}$ is also locally bounded on $\Omega^{\prime}$. Hence $\left\{\Phi^{n}\right\}$ is also locally bounded on $\Omega^{\prime}$. Therefore $\Omega=\Omega^{\prime}$. Hence $\Omega$ is pseudoconvex and even Runge.

We can take $S^{\prime}=S_{q_{0}}$.

We show next that all limits of subsequences of $\left\{\Phi^{n}\right\}$ have generic rank $r$. Assume $f=\lim \Phi^{n_{j}}$. We can assume that the limit $\tilde{f}:=\lim \Phi^{n_{j} i \ell}$ exists also on $\Omega^{\prime}$. By assumption the ranks of $f, f^{\ell}$ are $\leq r$. On the other hand on $S^{\prime}$ the rank is $r$, so the rank of $\tilde{f}$ is generically equal to $r$. Moreover, near points on $S_{q_{0}}$, $\tilde{f}=f^{i \ell}$ so generic rank of $f$ is $r$.

Remark 3.4 We have shown that recurrent Fatou components for $\Phi^{\ell}$ are Fatou components for $\Phi$. 
Proposition 3.5 Let $\Phi \in \mathscr{E}_{m}$. Assume $\Omega$ is a Fatou component for $\Phi$ with compact complement $K$. Then $\left(\Phi^{n}\right)$ diverges to infinity on $\Omega$.

Proof. We have $\Phi(\Omega) \subset \bar{\Omega}$ since $\Omega$ contains the complement of a ball. Since $\Phi(\Omega)$ is an unbounded set, we can choose $p \in \Omega$ so that $\Phi(p) \in \Omega$ also. Choose $U \subset \subset \Omega$, connected with $p$ and $\Phi(p)$ in $U$. Define $E:=\cap_{N} \overline{\cup_{n \geq N} \Phi^{n}(U)}$. Then $E$ is connected. Using Theorem 3.3 we know that $\Omega$ is not recurrent. Hence $E \subset K \cup \infty$. If $E \subset K$, then $\Omega$ must be Runge, which it is not, hence $E=\infty$.

Proposition 3.6 Let $\Omega$ be a Fatou component for $\Phi \in \mathscr{T}$. Then either $\Phi^{n}$ converges to infinity on $\Omega$ or $\Omega$ is recurrent and $\overline{\left\{\Phi_{\mid \Omega}^{n}\right\}}$ is a compact Lie Group in $\operatorname{Aut}(\Omega)$.

Proof. If $\Omega$ is not wandering, $\Omega$ is periodic, so for some $k \in \mathbb{Z}^{+}, \Phi^{k}(\Omega)=\Omega$. If $\Omega$ is not recurrent, then $\left\{\Phi_{\mid \Omega}^{k n}\right\}_{n} \rightarrow \partial \Omega \cup \infty$ uniformly on compact sets. Assume $\Phi^{n_{j} k}$ converges to a holomorphic map $h: \Omega \rightarrow \mathbb{C}^{k}$. Then $h(\Omega) \subset \partial \Omega$, so $h$ has rank at most $k-1$, contradicting that $\Phi$ is volume preserving. Hence $\Phi_{\mid \Omega}^{n k} \rightarrow \infty$ uniformly on compact sets. But then, also $\left\{\Phi_{\mid \Omega}^{n}\right\}$ converges to infinity.

Suppose next that $\Omega$ is wandering but that $\Phi^{n}$ does not converge to infinity. Then some subsequence $\left\{\Phi_{\mid \Omega}^{n_{i}}\right\}$ has a limit $F: \Omega \rightarrow \Omega^{\prime}:=f(\Omega) \subset \mathbb{C}^{k}$. But then $\Omega^{\prime}$ is an open set and since $\Phi$ is volume preserving, $\Omega$ is preperiodic, hence periodic. This contradicts that $\Omega$ is wandering.

\section{Isolated components in Julia sets}

An upper triangular automorphism of $\mathbb{C}^{k}$ is an automorphism of the form $\Phi\left(z_{1}, \cdots, z_{k}\right)=\left(\lambda_{1} z_{1}, \lambda_{2} z_{2}+h_{2}\left(z_{1}\right), \cdots, \lambda_{k} z_{k}+h_{k}\left(z_{1}, \cdots, z_{k-1}\right)\right.$ where $\lambda_{j} \in \mathbb{C}^{*}$ and each $h_{j}$ is an entire function.

Proposition 4.1 Let $\Phi \in \mathscr{B}$. Suppose that 0 is an isolated point in the Julia set $J_{\Phi}$. Then 0 is a repelling fixed point and $J_{\Phi}=\{0\}$. Moreover $\Phi$ is conjugate to an upper triangular map.

Proof. Pick a small ball $U$ containing 0 , and let $\Omega$ be a Fatou component containing $U \backslash\{0\}$. The sequence $\left(\Phi^{n}\right)$ is not locally bounded in $\Omega$. We can assume that $\partial U \subset \Omega$. Since $\{0\}$ is not in $\Omega$, there is a sequence $n_{j}$, and points $p_{j} \rightarrow 0$ with $\left\{\Phi^{n_{j}}\left(p_{j}\right)\right\}$ bounded and $\Phi_{\mid \partial U}^{n_{j}} \rightarrow \infty$. We may assume then that $\Phi^{n_{j}}(U) \supset \supset U$ for all $n_{j}$. Fix any $n_{j}$. Then there is a repelling fixed point $q$ for $\Phi^{n_{j}}$ in $U$. But since 0 is the only point of $J_{\Phi}$ in $U$, necessarily, $q=0$. Hence 0 is on a periodic orbit of some minimal order $\ell$ and hence $n_{j}=\ell m_{j}$. We observe that $\Phi^{-n_{j}}$ is contracting on $\Phi^{n_{j}}(U)$ and the iterates $\Phi^{m n_{j}}$ restricted to $\Phi^{n_{j}}(U)$ converge to 0 . If $n_{j}$ is large enough the whole orbit of 0 is contained in $\Phi^{n_{j}}(U)$. Hence there can be no other points on this orbit, so $\ell=1$ and 0 is a repelling fixed point for $\Phi$. By the invariance of the Fatou set, it also follows that 0 is the only point in the 
Julia set. Moreover, the basin of attraction of $\Phi^{-1}$ consists of all of $\mathbb{C}^{k}$. Using a classical result by ([RR]) we get that $\Phi^{-1}$ is conjugate to an upper triangular automorphism. The same is true for $\Phi$.

Theorem 4.2 Let $\Phi \in \mathscr{E}_{m}$ and suppose that the Julia set is not perfect. Then $\Phi$ is biholomorphic and conjugate to an upper triangular automorphism.

We need a preliminary result on fixed points.

Recall that $\omega$ is holomorphically homotopic to a point in $\Omega$ if there exists a continuous mapping $h:[0,1] \times \bar{\omega} \rightarrow \Omega$ with $h(1, z)=z$ and $h(0, z)=p$, where $p \in \omega$, moreover $h(t, \cdot)$ is holomorphic in $\omega$ for every $t \in[0,1]$.

Lemma 4.3 Let $\omega \subset \subset \Omega \subset \subset \mathbb{C}^{k}$ be connected open sets. Let $f: \bar{\omega} \rightarrow \Omega$ be a holomorphic map. Assume $\omega$ is holomorphically homotopic in $\Omega$ to a point $p \in f(\omega) \cap \omega$ and that $f(\partial \omega)$ is disjoint from $\cup_{0 \leq s \leq 1} h(s, \bar{\omega})$. Then $f$ has a fixed point in $\omega$.

Proof. We can assume that $p=0$. Define $f(s, z):=f(z)-h(s, z)$. Then $f(s, z)$ never vanishes on $[0,1] \times \partial \omega$. Since $f(0, \cdot)$ takes the value 0 on $\omega$, the same holds for $f(1, \cdot)$ i.e. the number of fixed points for $f$ is the same as the number of zeroes of $f(z)$ counted with multiplicity.

Remark 4.4 If $f$ is a biholomorphism in the above Lemma, then $f$ has a unique fixed point in $\omega$ and this fixed point is repelling. (In this case the existence of a unique fixed point can be proved directly using that $f^{-1}$ is strictly contracting for the Kobayashi metric.)

Proof of previous Theorem. Assume that $J_{\Phi} \cap B(0,2)=(0)$. If every converging subsequence of $\Phi^{n}$ has a finite limit on $A=\{1 / 2 \leq|z| \leq 1\}$, then $0 \notin J$. So there exists a subsequence $\Phi^{m_{j}}$ converging on $A$ to $\infty$ but not converging to $\infty$ on $\mathbb{B}(0,1)$. So, we can assume there is a sequence $p_{j} \rightarrow 0$ such that $\Phi^{m_{j}}\left(p_{j}\right) \rightarrow q \in \mathbb{C}^{k}$. Fix $R>>\|q\|$. Choose $m_{j}$ such that $\Phi^{m_{j}}(A) \cap B(0,2 R)=\emptyset$. Let $\omega_{j}$ be the connected component of $\Phi^{-m_{j}}(B(0, R))$ containing $p_{j}$ and contained in $B(0,1 / 2)$. Then $\Phi^{m_{j}}$ has a fixed point $x_{0}$ in $\omega_{j}$, by Lemma 4.3. If $x_{0} \neq 0$, then some sequence of iterates converges to $\infty$ contradicting that $x_{0}$ is periodic. Hence $x_{0}=0$, so 0 is periodic. For the same reason there is no point $x \neq 0$ in $\omega_{j}$ so that $\Phi^{m_{j}}(x)=0$, because some subsequence of $\Phi^{n}(x)$ tends to infinity, and 0 is periodic.

Let $\ell$ be the period of 0 . Then $m_{j}=n_{j} \ell$. Consider $\Phi^{m_{j}}: \omega_{j} \rightarrow B(0, R)$ for large $m_{j}$. Then $\Phi^{m_{j}}: \omega_{j} \rightarrow B(0, R)$ is a proper map and 0 cannot have a stable manifold, so $\Phi^{m_{j}{ }^{\prime}}(0)$ has no zero eigenvalue. Hence $\Phi^{m_{j}}$ is invertible near 0 , so the sheet number is one. Hence $\Phi^{m_{j}}$ is biholomorphic on $\omega_{j}$. Then $\Phi^{m_{j}-r l}$ is injective on $\omega_{j}$ and $\Phi^{r \ell}: \Phi^{m_{j}-r \ell}\left(\omega_{j}\right) \rightarrow B(0, R)$ is biholomorphic. Hence $\Phi^{-r \ell}$ is well defined on $B(0, R)$ with $\Phi^{-r \ell}(0)=0$. Since $R$ is arbitrary, $\Phi^{-r \ell}$ is globally defined with $\Phi^{-r \ell}: \mathbb{C}^{k} \rightarrow \Omega$ is biholomorphic for some $\Omega \subset \mathbb{C}^{k}$.

Suppose $\partial \Omega \neq \emptyset$. Pick $p \in \partial \Omega$ and $\left(p_{n}\right) \subset \Omega, p_{n} \rightarrow p, p_{n}=\left(\Phi^{-\ell}\left(q_{n}\right)\right)$. The $\left(q_{n}\right)$ cannot have a finite limit point. Then $\Phi^{\ell}\left(p_{n}\right) \rightarrow \infty$ which is impossible. Hence $\Phi^{-\ell}$ is a global biholomorphism. So $\Phi$ is biholomorphic. 
Proposition 4.1 implies that $\Phi$ is conjugate to a triangular mapping.

Remark 4.5 Theorem 4.2 shows in particular that when $k=1$ and $\Phi$ is not linear, the Julia set is perfect. The proof does not use the theory of periodic points.

\section{Maps with empty Julia set}

Theorem 5.1 Let $\Phi \in \mathscr{E}_{m}$. Assume the Julia set of $\Phi$ is empty. Then either $\left(\Phi^{n}\right)$ diverges to infinity on $\mathbb{C}^{k}$ or there is a closed analytic submanifold $S \subset \mathbb{C}^{k}$ with the following properties:

(0) $\Phi_{\mid S} \in \operatorname{Aut}(S)$,

(1) $S$ is holomorphically trivial, i.e. there is a continuous family of holomorphic maps $\tilde{h}_{t}: S \rightarrow S, t \in[0,1], \tilde{h}_{1}=I d$ and $\tilde{h}_{0} \equiv$ constant,

(2) The images of $\left\{\Phi^{n}\right\}$ converge uniformly on compact sets to $S$.

(3) $\overline{\left\{\Phi_{\mid S}^{n}\right\}}$ is isomorphic to $T^{\ell} \times A$ where $T^{\ell}$ is a torus of dimension $\ell$ and $A$ is a finite group.

Proof. Assume $\left(\Phi^{n}\right)$ does not converge uniformly on compact sets to infinity. Then $\mathbb{C}^{k}$ is a recurrent Fatou component and $\left(\Phi^{n}\right)$ is locally bounded as follows from Theorem 3.3. Let $h=\lim \Phi^{n_{i}}$ have maximal generic rank $r$ Since $\Phi^{n_{i+1}-n_{i}}\left(\Phi^{n_{i}}\right)=\Phi^{n_{i+1}}$ we can construct $\tilde{h}=\lim \Phi^{m_{i}}$ such that $\tilde{h} \circ h=h$. Here $\left\{m_{i}\right\}$ is a subsequence of $\left\{n_{i+1}-n_{i}\right\}$. Hence $\tilde{h}=\operatorname{Id}$ on $h\left(\mathbb{C}^{k}\right)$. Let $S=\{q ; \tilde{h}(q)=q\}$. Then $S$ is a subvariety in $\mathbb{C}^{k}$ containing $h\left(\mathbb{C}^{k}\right)$. Let $S_{1}$ be the irreducible branch of $S$ containing $h\left(\mathbb{C}^{k}\right)$. Then $S_{1}$ is a manifold of dimension $r$ and is isolated in $S$. We show that $\tilde{h}\left(\mathbb{C}^{k}\right)=S_{1}$. Let $U=\tilde{h}^{-1}\left(S_{1}\right)$. Then $U$ is an open set containing $S_{1}$. If $p \in \partial U$, then $\tilde{h}(p) \in S_{1}$, so $U=\mathbb{C}^{k}$. Hence $S=S_{1}$. One shows easily that $\Phi(S) \subset S$ and since $\Phi_{\mid S}^{m_{i}} \rightarrow$ Id it follows that $\Phi$ is an automorphism of $S$.

As in the proof of Theorem 3.3 one shows that $\overline{\left\{\Phi_{\mid S}^{n}\right\}} \cap \mathrm{A} u t(S)$ is a compact subgroup $G$ of $\operatorname{Aut}(S)$.

If $V$ is a bounded open set in $S$, then $\tilde{V}=\cup_{n} \Phi^{n}(V)$ is also a bounded open set in $S$ and $\Phi$ is an automorphism of $\tilde{V}$. Hence $G$ is a commutative compact Lie group, see ([Ko]) p.70 or ([Na]), which is therefore isomorphic to $T^{\ell} \times A$ where $A$ is a finite commutative group.

It follows that $\overline{\left\{\Phi_{\mid S}^{n}\right\}}$ is contained in $\mathrm{A} u t(S)$. [Let $\Phi^{k_{i}}$ be a convergent subsequence, converging to $\psi$, writing $\Phi^{m_{j(i)}}=\Phi^{m_{j(i)}-k_{i}} \circ \Phi^{k_{i}}$ for $j(i)$ large, we see that $\psi \in \operatorname{Aut}(S)$.] Assume $\Phi^{\ell_{i}} \rightarrow h_{1}$, then $h_{1}\left(\mathbb{C}^{k}\right) \supset S$ and by the above argument $h_{1}\left(\mathbb{C}^{k}\right)=S$. So property (2) is proved. Since $\tilde{h}$ is a holomorphic retraction on $S$, it follows that $S$ is holomorphically trivial, i.e. $\tilde{h_{t}}(z):=\tilde{h}(t z)$ then $\tilde{h_{t}}$ joins the identity to the constant map as $t$ varies from 1 to 0 . 


\section{Oscillating Fatou components}

Definition 6.1 Let $\Omega$ be a Fatou component for $\Phi \in \mathscr{E}_{m}$. Then $\Omega$ is oscillating if some subsequence of iterates is locally uniformly bounded on $\Omega$ and some other subsequence converges u.c.c. to infinity.

Theorem 6.2 There exists a biholomorphic map $\Phi$ of $\mathbb{C}^{2}$ which has a wandering oscillating Fatou component.

To find a $\Phi$ we will use the concept of an $r$-shift. This consists of a sequence of regions converging to infinity and a shift between them. Let $0<r<1 / 2$.

Definition 6.3 $A(\Psi, r)$ - shift $X=X\left(\Psi, r,\left\{\Omega_{i}\right\}\right)$ is a biholomorphic map $\Psi$ of $\mathbb{C}^{2}$ together with a sequence of regions $\left\{\Omega_{i}\right\}_{i=-\infty}^{\infty}$ where $\Delta\left((2 i, 0) ; r-r^{2}\right) \subset$ $\Omega_{i} \subset \Delta\left((2 i, 0) ; r+r^{2}\right), \Psi: \Omega_{i} \rightarrow \Omega_{i+1}$ and $\Psi-(z+2, w)$ vanishes at each center point $(2 i, 0)$.

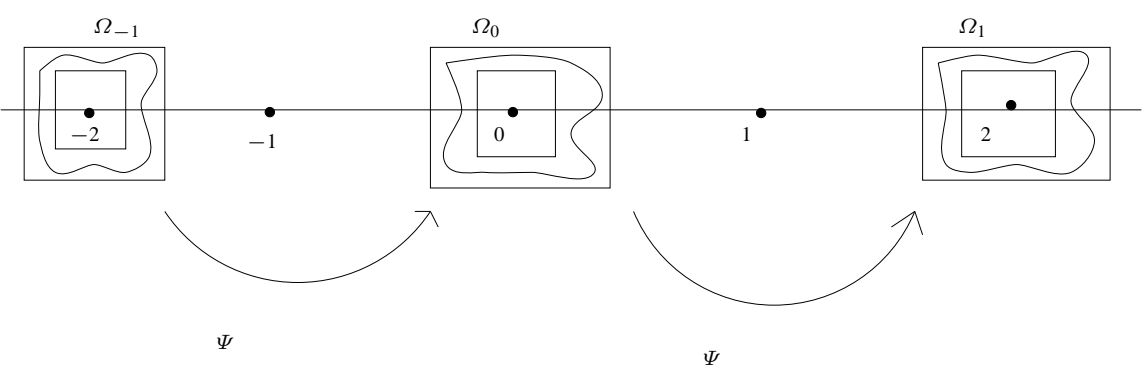

Fig. 1. A $(\Psi, r)-$ shift

We will inductively glue almost oscillating domains to $r$ - shifts in such a way that in the limit one has an oscillating domain.

\section{Definition 6.4 $A n$ almost oscillating $\Psi$ - domain}

$$
Y=Y\left(\Psi,\left\{U_{i}\right\},\left\{D_{i}\right\},\left\{r_{i}\right\},\left\{s_{i}\right\}\right)
$$

consists of a sequence of domains $\left\{U_{i}\right\}_{i=-\infty}^{\infty}$, polydiscs $D_{i}, \quad U_{i} \subset D_{i}:=$ $\Delta\left(z_{i} ; r_{i}\right) \times \Delta\left(w_{i}, s_{i}\right)$ for which the $\overline{\Delta\left(z_{i} ; r_{i}\right)}$ is a locally finite pairwise disjoint family and there is a biholomorphic map $\Psi$ of $\mathbb{C}^{2}$ for which $\Psi\left(U_{i}\right)=U_{i+1} \forall i$. We assume $(1,0) \in U_{0}$.

It follows that $\lim z_{i}=\infty$. 


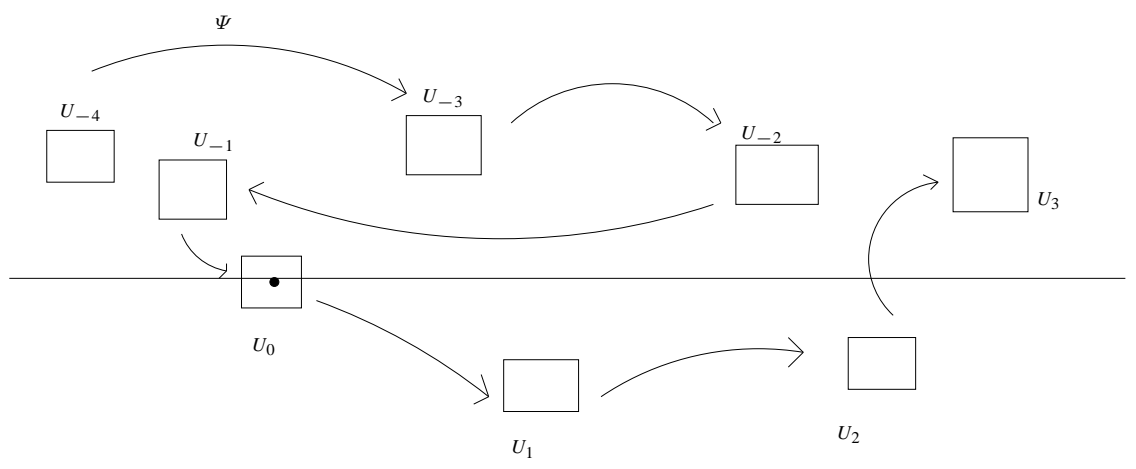

Fig. 2. Almost oscillating $\Psi-$ domain

We say that shifts and almost oscillating domains are disjoint if they project disjointedly on the $z$ - axis.

Definition 6.5 We say that a $(\Psi, r)$ - shift and an almost oscillating $\Psi$ - domain are disjoint if the discs $\overline{\Delta\left(2 i ; r+r^{2}\right)}, \overline{\Delta\left(z_{j} ; r_{j}\right)}$ are all pairwise disjoint. domains.

We will define a gluing of disjoint $(\Psi, r)$ - shifts and almost oscillating $\Psi-$

Definition 6.6 Given a $(\Psi, r)-\operatorname{shift} X\left(\Psi, r,\left\{\Omega_{i}\right\}\right)$ and an almost oscillating $\Psi-$ domain $Y\left(\Psi,\left\{U_{i}\right\},\left\{D_{i}\right\},\left\{r_{i}\right\},\left\{s_{i}\right\}\right)$ which are disjoint, and $R>0$, an $R$ - gluing is an almost oscillating $\Phi$-domain $Y^{\prime}\left(\Phi,\left\{U_{i}^{\prime}\right\},\left\{D_{i}^{\prime}\right\},\left\{r_{i}^{\prime}\right\},\left\{s_{i}^{\prime}\right\}\right)$ with the following properties:

(i) $\|\Phi-\Psi\| \leq 1 / R$ when $\|(z, w)\| \leq R$,

(ii) $U_{0}^{\prime}=U_{0}$,

(iii) Let $i_{0}$ be the smallest integer such that $\left|z_{k}\right|-r_{k}>R \forall k \geq i_{0}$. Then $U_{i}^{\prime} \subset \Delta\left(\left(2 i-2 i_{0}-2[R]-2,0\right) ; r+2 r^{2}\right) \forall i \geq i_{0}$.

Condition (iii) is crucial. It means that $U_{i}^{\prime}$ comes back before going to infinity. In our construction $U_{i}^{\prime}$ will be close to an edge of the polydisc. 


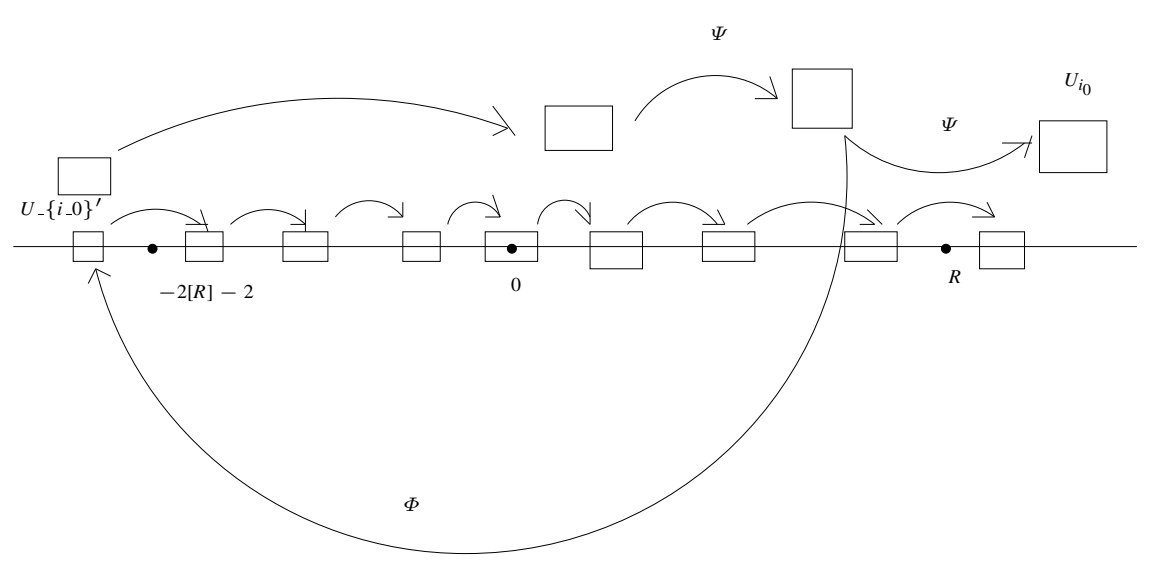

Fig. 3. Gluing

Lemma 6.7 Any $(\Psi, r)-$ shift and any almost oscillating $\Psi$ - domain which are disjoint have an $R-$ gluing for any $R$. The $R-$ gluing is disjoint from some $r^{\prime}-$ shift.

Proof. To obtain an $R$ - gluing, we compose $\Psi$ with a suitable choice of maps. We want to map $U_{i}$ into $\Omega_{i-i_{0}-[R]-1}$ for $i \geq i_{0}$.

Remark first that we can assume that $\left|w_{i}\right|+\left|s_{i}\right|$ are arbitrarily small when $|i| \rightarrow \infty$. Simply compose $\Psi$ with a map of the form

$$
(z, w) \rightarrow\left(z, w e^{\phi(z)}\right),
$$

where $\phi(z)$ is an entire function which is arbitrarily close to 0 on $\cup \Delta(2 i, r+$ $r^{2}$ ) and on finitely many $\Delta\left(z_{i}, r_{i}\right)$ while $\Re \phi(z)_{\mid \Delta\left(z_{i}, r_{i}\right)} \rightarrow \infty$ when $i \rightarrow-\infty$, $\Re \phi(z)_{\mid \Delta\left(z_{i}, r_{i}\right)} \rightarrow-\infty$ when $i \rightarrow \infty$. We also want $\phi(z)$ to vanish at each $z=2 i$.

Let $\Psi_{1}$ be a map of the same form, $\Psi_{1}(z, w)=\left(z, w e^{\phi_{1}(z)}\right)$ where $\phi_{1}$ is close to 0 on $\Delta(0, R+1) \cup \cup_{i} \Delta\left(z_{i}, r_{i}\right)$ and $\Re \phi_{1} \Omega_{i} \rightarrow \infty$ as $i \rightarrow-\infty$. In this way we arrange that the $\Omega_{i}$ 's are flattened arbitrarily fast at infinity in the $w$ - direction during the proof and at the end we compose again with a similar kind of map to restore the new $\Omega_{i}$ back to the correct essentially $i$ - independent height.

To glue, we will move $U_{i_{0}}$ into $\Omega_{-[R]-1}$ and also contracting $U_{i_{0}}$ to an arbitrarily small subset - $U_{i_{0}}^{\prime}$ will be a small perturbation of this set - containing the point $(-2[R]-2-r / 2,0)$ i.e. far from the center of $\Omega_{-[R]-1}$. We move $U_{i_{0}}$ in three steps. 


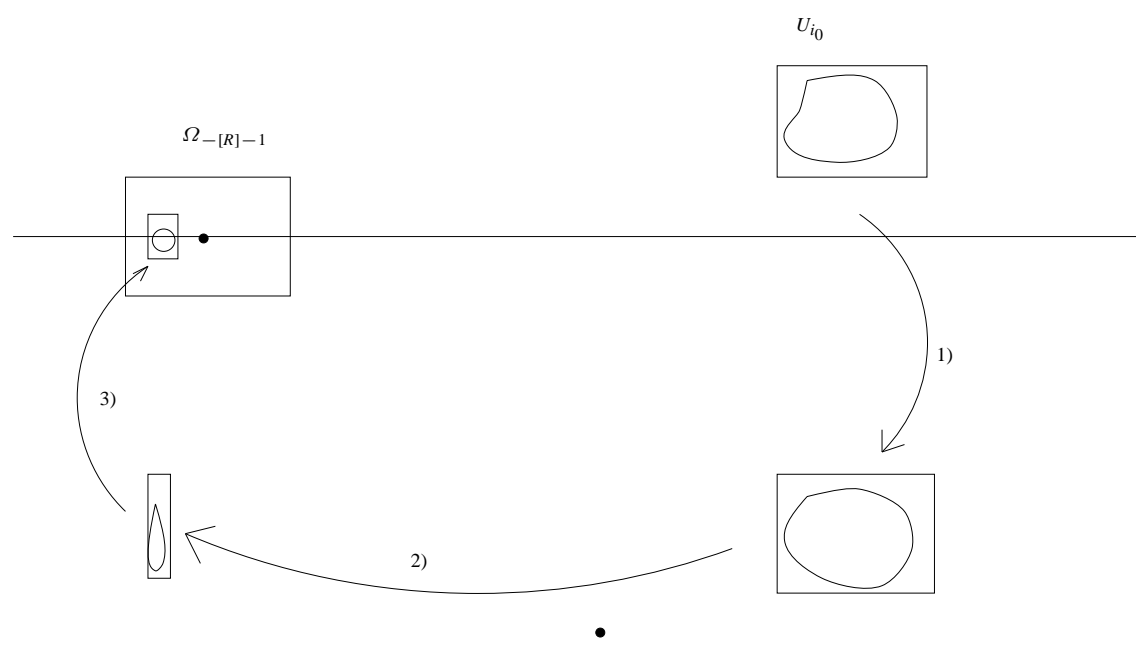

Fig. 4. Inserting $U_{i_{0}}$ into $\Omega_{-[R]-1}$

1) Compose with a map of the form

$$
(z, w) \rightarrow(z, w+\phi(z)) .
$$

Here $\phi(z)$ is close to zero on all discs $\Delta\left(2 i, r+r^{2}\right), \Delta\left(z_{j}, r_{j}\right)$ and $\Delta(R, 0)$ except $\overline{\Delta\left(z_{i_{0}}, r_{i_{0}}\right)}$ where $|\phi(z)|>>R$ and is almost constant. Moreover, $\phi(z)$ vanishes at each center $2 i$. Notice that replacing $\Psi$ with the composition with this map we still have disjoint $(\Psi, r)$ - shift and oscillating $\Psi$ - domain if we only consider $\Delta\left(z_{j}, r_{j}\right)$ for $j \leq i_{0}$. We will continue our construction so as also to include the indices $j>i_{0}$.

2) We next compose with a map which will contract (the new) $U_{i_{0}}$ in the $z-$ direction and move $U_{i_{0}}$ to the right location in the $z$ - direction. We compose with a map of the form

$$
\left(e^{g(w)} z-h(w), w\right)
$$

Here $g$ vanishes at 0 and is arbitrarily small on $\Delta(0, R+1)$. In order to compress $U_{i_{0}}$ in the $z$-direction we make $g$ arbitrarily close to a large negative constant on $U_{i_{0}}$.

Finally, we move $U_{i_{0}}$ to a $z$ - coordinate close to $-2[R]-2-r / 2$ by letting $h(w)$ have an appropriate almost constant value on $U_{i_{0}}$ while $h$ vanishes at 0 and is arbitrarily small on $\Delta(0, R+1)$, this is where we use that $\left|w_{i}\right|+\left|s_{i}\right| \rightarrow 0$ arbitrarily fast and $\Omega_{i}$ are flattened in the $w$-direction. So $g, h$ are arbitrarily close to 0 on $\Omega_{i}$ thereby preserving the $r$-shift.

3) Next we insert $U_{i_{0}}$ into $\Delta\left((-2[R]-2,0) ; r+r^{2}\right)$ by composing with a map of the form

$$
\left(z, e^{G(z)} w+H(z)\right)
$$


Here $G(z), H(z)$ are arbitrarily close to zero on all discs $\Delta\left(2 i ; r+r^{2}\right), i \neq$ $-[R]-1, \Delta(-2[R]-2 ; r / 4), \Delta\left(z_{j} ; r_{j}\right)$ and $\Delta(0 ; R+1)$ and vanishes at all centers. Moreover $G(z)$ is arbitrarily close to a large negative constant on $\Delta(-2[R]-$ $2-r / 2 ; r / 8)$ in order to compress $U_{i_{0}}$ in the $w$-direction while $H(z)$ has a suitable, almost constant value to move $U_{i_{0}}$ to the desired location.

We next let $\Phi$ denote the composition of these three maps and $\Psi$ and define $U_{0}^{\prime}:=U_{0}, U_{i}^{\prime}:=\Phi^{i}\left(U_{0}^{\prime}\right)$. This defines an almost oscillating $\Phi-$ domain. Moreover $\Phi$ defines a $\left(\Phi, \frac{r}{8}\right)-$ shift which is disjoint.

Proof of the Theorem. To prove the Theorem, one uses an inductively defined seqence of gluings and pass to a limit. The first step of the inductive construction, is to use the map $\Psi(z, w)=(z+2, w)$. Also we define a $(\Psi, 1 / 4)-$ shift by letting $\Omega_{i}:=\Delta((2 i, 0) ; 1 / 4)$ Our first almost oscillating $\Psi-$ domain consists of the domains $U_{i}:=\Delta((2 i+1,0) ; 1 / 4)$.

If we choose $R_{n} \rightarrow \infty$ fast enough, then after passing to the limit in the inductive construction we obtain finally an almost oscillating $\Phi$ domain which consists of domains $\left\{U_{i}^{\prime}\right\}$ and the forward iterates $\Phi\left(U_{i}^{\prime}\right)$ pass infinitely often close to the sequence $(2 j, 0)$ and to $U_{0}$ and so is oscillating.

Let $\Omega$ be the Fatou component containing $U_{0}^{\prime}$. We know completely the orbit of $U_{0}^{\prime}$. It remains to show that $\Omega$ is wandering. If not, there is an integer $k \geq 1$ such that $\Phi^{k}(\Omega)=\Omega$. Let $\gamma$ be a curve contained in $\Omega$ connecting $U_{0}^{\prime}$ to $U_{k}^{\prime}$. Also let $U$ be a connected neighborhood of $\gamma$ contained in $\Omega$. For high iterates $n_{j}, \Phi_{\mid U_{0}^{\prime} \cup U_{k}^{\prime} \cup U}^{n_{j} k}$ converges u.c.c. to $(0,0)$ on $U_{0}^{\prime}$ and to $(2 k, 0)$ on $U_{k}^{\prime}$. This is a contradiction.

\section{References}

[Be] Bergweiler, W; Iteration of meromorphic functions, Bull. A. M. S. 29, (1993), 151-188.

[Bo] Bourbaki, N; Theories Spectrales, Herman, Paris.

[B] Buzzard, G; Kupka-Smale Theorem for Aut $\left(\mathbb{C}^{n}\right)$, preprint 1994.

[FS1] Fornæss, J. E., Sibony, N; Classification of recurrent domains for some holomorphic maps, to appear Math. Ann.

[FS2] Fornæss, J. E., Sibony, N; Holomorphic Symplectomorphisms in $\mathbb{C}^{2}$, World Sc. Ser. Appl. Anal., Vol. 4, Dynamical Systems and Applications, July 1995.

[FS3] Fornæss, J. E., Sibony, N; Holomorphic symplectomorphisms in $\mathbb{C}^{2 p}$, Duke Math. Journal 1995.

[FS4] Fornæss, J. E., Sibony, N; The Closing Lemma for Holomorphic Maps, preprint 1995.

[FM] Friedland, S., Milnor, J; Dynamical Properties of plane automorphisms, Ergodic Theory and Dynamical Systems 9, (1989), 67-99.

[G] Gunning, R. C; Introduction to holomorphic functions of several variables II, The Wadsworth $\&$ Brooks/Cole Mathematics Series, (1990).

[Ho] L. Hörmander; An introduction to Complex Analysis in Several Variables, North Holland, Amsterdam, (1973).

[Ko] Kobayashi, S; Hyperbolic manifolds, Marcel Dekker, New York 1970.

[Na] Narasimhan, R; Several complex variables, University of Chicago Press, (1971).

[RR] Rosay, J. P, Rudin, W; Holomorphic maps from $\mathbb{C}^{n}$ to $\mathbb{C}^{n}$, TAMS 310 (1988), 47-86.

[W] Wermer, J; Function Algebras and Several Complex Variables, Berlin-Heidelberg-New York, Springer, (1976). 